\title{
Lung Volume Changes, Occlusion Pressure and Chest Wall Configuration in Human Infants
}

\author{
ANN R. STARK, (31) MICHAEL D. GOLDMAN, AND IVAN D. FRANTZ, III \\ Department of Pediatrics, Harvard Medical School, Department of Physiology, Harvard School of Public Health, and \\ Boston Hospital for Women, Boston, Massachussetts, USA
}

\section{Summary}

Measurements of respiration were compared in normal, full term and premature infants before and after application of $5 \mathrm{~cm}$ $\mathrm{H}_{2} \mathrm{O}$ continuous negative pressure (CNEG) around the body below the neck. Mean minute ventilation in the full term infants decreased when CNEG was applied, secondary to a decrease in respiratory rate, with tidal volume relatively unchanged. The premature infants showed no consistent ventilatory response to CNEG. There were variable changes in occlusion pressure at increased lung volume. Observation with rib cage and abdomen anterior-posterior (A-P) magnetometers showed that the increase in end-expiratory lung volume was accounted for largely by expansion of the rib cage so that the configuration of the diaphragm and its mechanical advantage were maintained. These results suggest that the diaphragm is the major determinant of mask occlusion pressure in infants.

\section{Speculation}

The effects of increased lung volume on the configuration of the chest wall may relate to the success of continuous distending pressure in the treatment of respiratory disorders in infants, including apnea of prematurity.

The pressure generated by the inspiratory muscles when the airway is occluded at end-expiration has been used as an index of the output of the respiratory center in adults (28), children (4), and newborn infants (8). No flow or volume changes occur after occlusion, except for a negligible amount due to decompression of thoracic gas. The occluded inspiratory pressure is independent of pulmonary compliance or resistance, hence, its proposed usefulness as an index of respiratory center output in place of the conventional index, minute ventilation. The pressure developed at a given level of neural drive depends on the mechanical configuration of the inspiratory muscles $(19,22)$. Thus, changes in resting lung volume that change the mechanical configuration of the inspiratory muscles may affect occlusion pressure.

Changes in lung volume are known to affect respiration in infants. Continuous distending pressure has been shown to abolish periodic breathing (27) and to decrease the frequency of apneic spells in premature infants (16). The mechanism of these changes may be related to changes in neuromuscular activity, blood gases, or other factors. The effect of increased lung volume on mask occlusion pressure and on the mechanical configuration of the chest wall in newborn infants has not been described. We have investigated the effect of increasing lung volume on mask occlusion pressure, tidal volume, frequency, and minute ventilation in newborn infants. In addition, we have observed with magnetometers the configurational changes of the thorax and abdomen when lung volume is increased, and have considered the effect of these changes on the measurement of occlusion pressure.

\section{MATERIALS AND METHODS}

Fifteen normal, full term infants (birth weight: $3.40 \pm 0.35 \mathrm{~kg}$, mean \pm SD) and seven healthy, premature infants of 32-34 wk gestation (birth weight: $1.70 \pm 0.20 \mathrm{~kg}$ ) were studied during the first 3 days of life. Informed consent for the study was obtained from the parents and pediatrician of each infant. Measurements were made after a feeding, with the infants sleeping quietly in the supine position, enclosed below the neck in a negative pressure box. In the premature infants, skin temperature was maintained at $36.5^{\circ}$ with a servocontrolled radiant warmer. Flow was measured with a heated Fleisch pneumotachograph (no. 00) and Statham PM 15E pressure transducer while the infant breathed through a face mask, and flow was integrated elecronically to give volume. Mask pressure was measured with a Hewlett-Packard 270 transducer. The dead space of the system was approximately $6 \mathrm{ml}$. All signals were recorded on a Beckman polygraph.

Measurements of tidal volume, respiratory frequency, minute ventilation, and mask occlusion pressure were compared for each infant before and after application of $5 \mathrm{~cm} \mathrm{H} \mathrm{H}_{2} \mathrm{O}$ CNEG. Respiratory frequency and minute ventilation were calculated from integrated flow measurements during a $1 \mathrm{~min}$ period of regular breathing at the start and end of the control period and of CNEG. Ten single-breath occlusions of the airway were done at endexpiration in each infant before and after the application of distending pressure, allowing a 30-60 sec recovery period between occlusions. The maximum inspiratory pressure $\left(\mathrm{P}_{\mathrm{m}}\right)$ and the pressure $100 \mathrm{msec}$ after occlusion $\left(\mathrm{P}_{.1}\right)$ were measured. The increment in lung volume that occurred with application of CNEG was measured with the pneumotachograph when the new resting volume was achieved, usually after 2-3 breaths. All measurements were made during quiet, regular breathing.

In three of the premature and eight of the full term infants, movement of the rib cage and abdomen in the A-P dimension was recorded with magnetometers (Source: N. H. Peterson, Harvard School of Public Health) placed in the midline at the upper onethird of the sternum and $1 \mathrm{~cm}$ above the umbilicus on anterior and posterior surfaces. These were the positions of maximal motion during quiet breathing and during changes in lung volume, as determined by visual inspection. In two of the full term infants, the lateral dimension of the rib cage was also recorded with magnetometers during changes in lung volume.

\section{RESULTS}

Tidal volume, respiratory rate, minute ventilation, and lung volume change during control breathing and after application of $5 \mathrm{~cm} \mathrm{H}_{2} \mathrm{O}$ CNEG are shown in Tables 1 and 2 . The mean increment in end-expiratory lung volume with the application of CNEG was $17.5 \mathrm{ml}$ (range $9-32 \mathrm{ml}$ ) in the full term infants. Lung volume increased an average of $7.7 \mathrm{ml}$ (range $5.8-10.8 \mathrm{ml}$ ) in the premature infants. Mean minute ventilation in the full term infants decreased when continuous distending pressure was applied $(P<$ $.05)$, secondary to a decrease in respiratory rate, with tidal volume 
Table 1. Respiratory measurements in full term infants

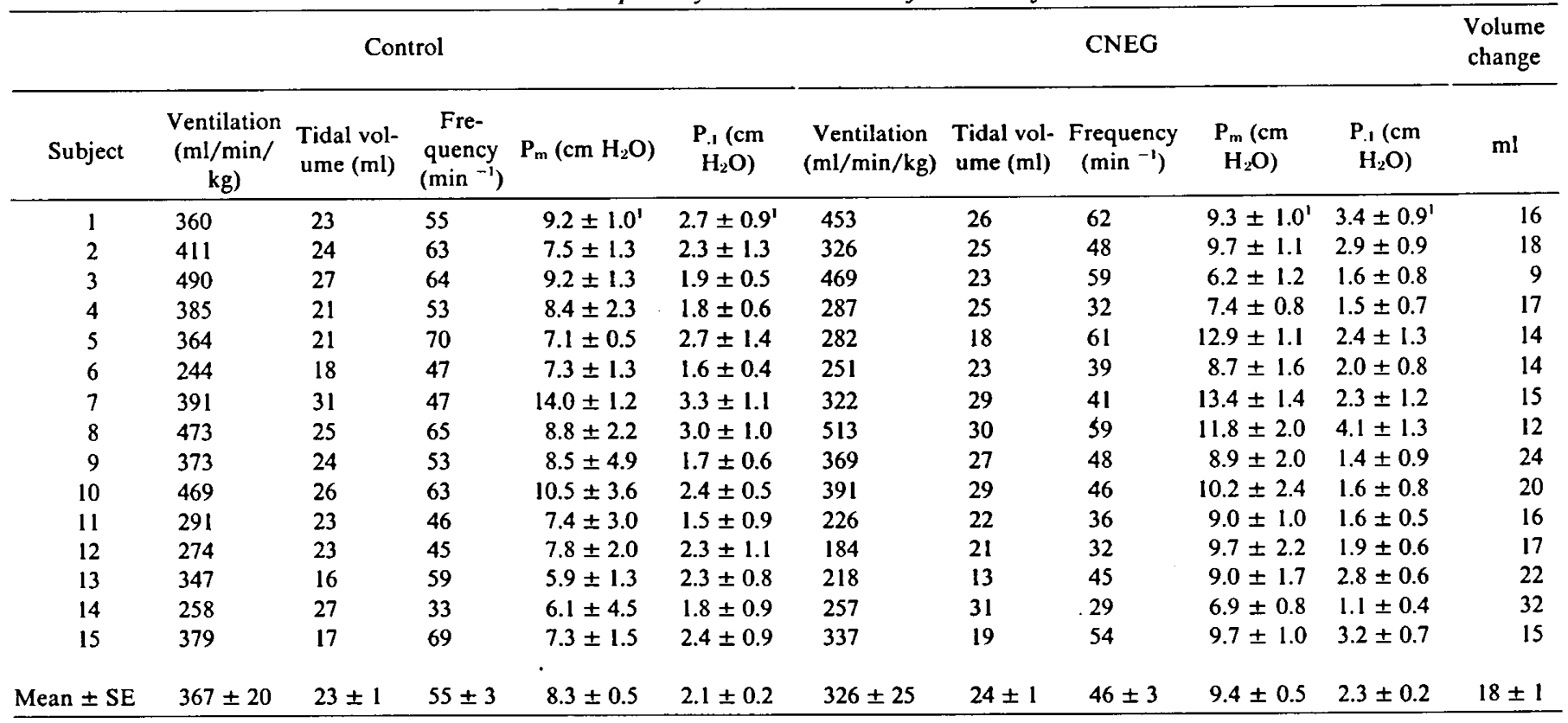

Table 2. Respiratory measurements in premature infants

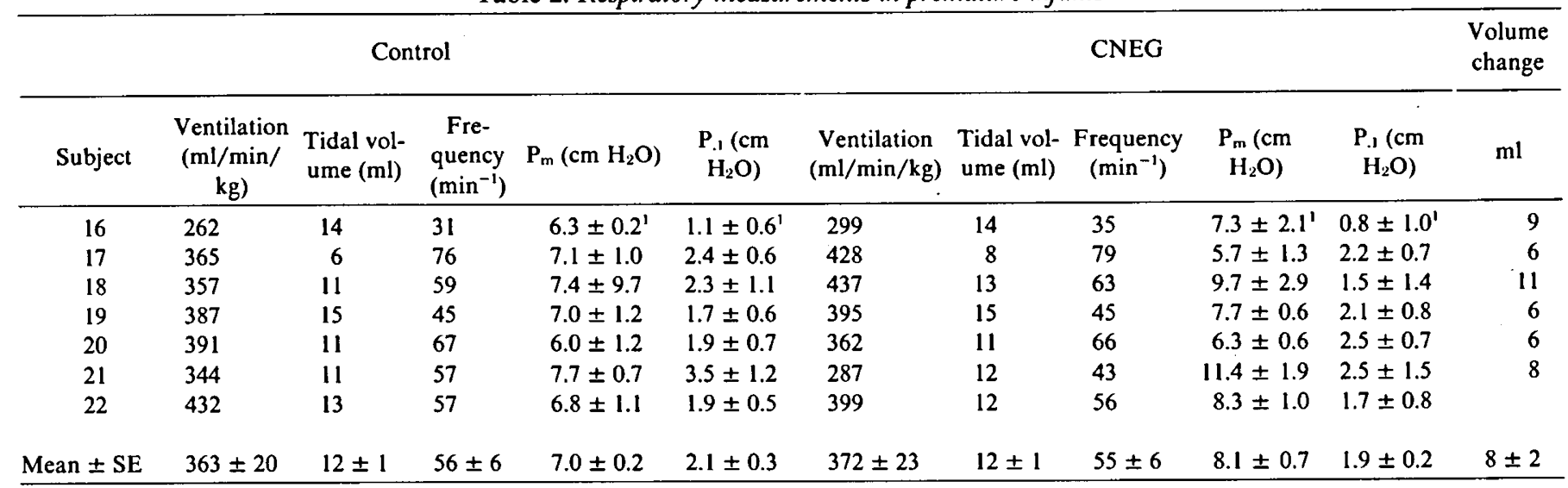

'Mean \pm SD.

relatively unchanged. This decrease in respiratory rate was secondary to prolongation of expiratory time. The premature infants showed no consistent ventilatory response to CNEG; minute ventilation increased in three, decreased in three, and remained unchanged in one infant.

$P_{m}$ and $P_{.1}$ before and after application of CNEG is shown for individual full term and premature infants in Tables 1 and 2. Each value is the average of at least 10 measurements made on each infant during control and CNEG. However, there was substantial variability within the measurements on most infants, both during control and CNEG, and none of the changes achieved statistical significance. Furthermore, while the $P_{m}$ increased in some infants during CNEG, the $P_{.1}$ in some of these individuals decreased on CNEG. The magnitude of change in $P_{m}$ did not correlate with the magnitude of change in lung volume.

Observations of the infants indicated that lung volume changes during CNEG were associated with large changes in the A-P diameter of the rib cage, but little change in the lateral dimension. This observation was confirmed in the infants in whom lateral magnetometer measurements were made. Thus, A-P measurements were used to assess rib cage movement during lung volume changes.
Occluded inspiratory efforts produce isovolume displacements of the rib cage and abdominal walls. Theoretically these maneuvers can be used to calculate the change in volume of the rib cage and abdomen during quiet breathing. In our studies, however, the thoraco-abdominal configuration changed during quiet breathing associated with movement of the infant, as did the relationship between rib cage and abdominal movement during airway occlusion. Thus, isovolume maneuvers performed at one point in the study could not be used to assess changes in rib cage and abdomen volume displacements quantitatively at a later time, but a qualitative evaluation could be made.

An example of a tidal volume breath and an end-expiratory occlusion during control breathing in a premature infant is displayed in an X-Y recording of rib cage and abdominal magnetometers in Figure 1. Paradoxical movement occurs during the initial part of inspiration, with the diameter of the abdomen increasing and the rib cage decreasing. The occluded inspiratory effort displaced the abdomen outward and the rib cage inward, resulting in a typical isovolume maneuver. Note that the abdominal diameter is similar at end-expiration of both the occluded and nonoccluded breaths.

Figures 2 and 3 show examples of increasing lung volume on 


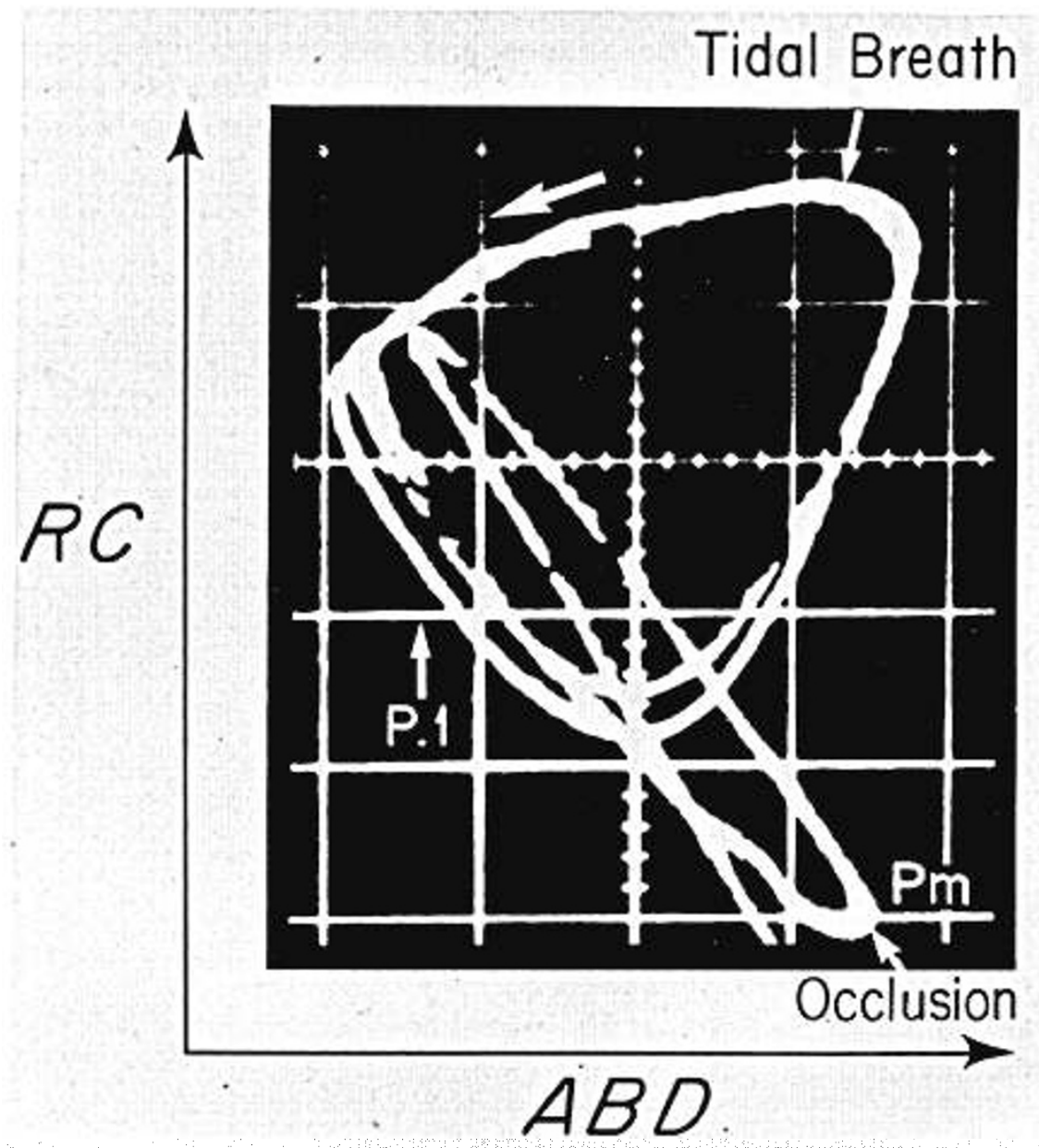

Fig. 1. Recording of rib cage (RC) and abdominal (ABD) A-P magnetometers during a tidal breath and during an occlusion at end-expiration. The arrows indicate the direction of increasing diameter. The loop is counter clockwise.

$\mathrm{X}-\mathrm{Y}$ recordings of rib cage and abdomen $\mathrm{A}-\mathrm{P}$ magnetometers in a full term and a premature infant. The tracings show tidal volume loops during control breathing and at increased lung volume. After the initial breaths on CNEG, the end-expiratory diameter of the abdomen was unchanged and rib cage expansion accounted for the increase in end-expiratory lung volume. The changes in lung volume and rib cage and abdominal diameters with continuous negative pressure are illustrated in Figure 4. When the endexpiratory lung volume increased by a volume approximately equivalent to one tidal volume, the rib cage diameter increased dramatically at end-expiration and end-inspiration, but the abdominal diameter changed little. In all infants studied, changes in the rib cage diameter accounted for most, or all, of the lung volume increment during CNEG.

Isovolume maneuvers performed during a control period and while on CNEG are shown in Figure 5. The two occlusions shown are the last one before increasing lung volume, and the first one after the increase. Intervening breaths are omitted for clarity, but the relative positions of the magnetometer traces are correct. Note that as in Figures 4 and 5, most of the volume change with CNEG is accounted for by rib cage expansion. Note also that the abdominal diameter is similar at all points during inspiration, both on and off CNEG and during tidal breaths and isovolume maneuvers. This relationship was seen consistently throughout the studies in all infants, and indicates that the configuration of the diaphragm was similar where $P_{.1}$ and $P_{m}$ were measured both at resting and increased lung volume.

\section{DISCUSSION}

We used magnetometers to record abdominothoracic displacements as outlined by Konno and Mead (18). In the present studies, direct visual inspection indicated that a substantial portion of the anterior thorax moved as a unit during breathing. The lateral thoracic walls were observed to undergo only very small displacements. This was the case in all infants studied, premature as well as full term, during quiet breathing under control conditions and with CNEG, and was documented in those infants in whom we obtained magnetometer recordings. We placed the magnetometers in an area where thoracic motion appeared relatively uniform. The magnetometer signals we recorded in these infants are representative of thoracic displacements observed during breathing both with and without CNEG.

In contrast to the movements of the rib cage, those of the abdomen appeared to show a consistent relationship between different parts of the anterior and lateral wall both during occlusion and during breathing. Furthermore, because the abdominal contents act as an hydraulic coupling between the diaphragm and abdominal wall, we expect abdominal displacements to reflect those of the diaphragm. Goldman and coworkers $(12,13,14)$ have reported that changes in diaphragmatic length are reflected more directly in abdominal displacements than in changes of lung volume or rib cage displacements.

We have noted above that during a study, movements of the infant sometimes produced a change in thoraco-abdominal configuration. In addition, the relationship between rib cage and abdominal movements during occluded inspiratory efforts, while usually showing the reciprocal changes seen in Figures 1 and 5 ("isovolume maneuvers") occasionally departed substantially from the characteristic isovolume lines. These departures were generally loop-shaped and appeared more like a spontaneous breath than an occluded inspiratory effort. Similar behavior was observed in some infants by Frantz et al. (7) and, although not 


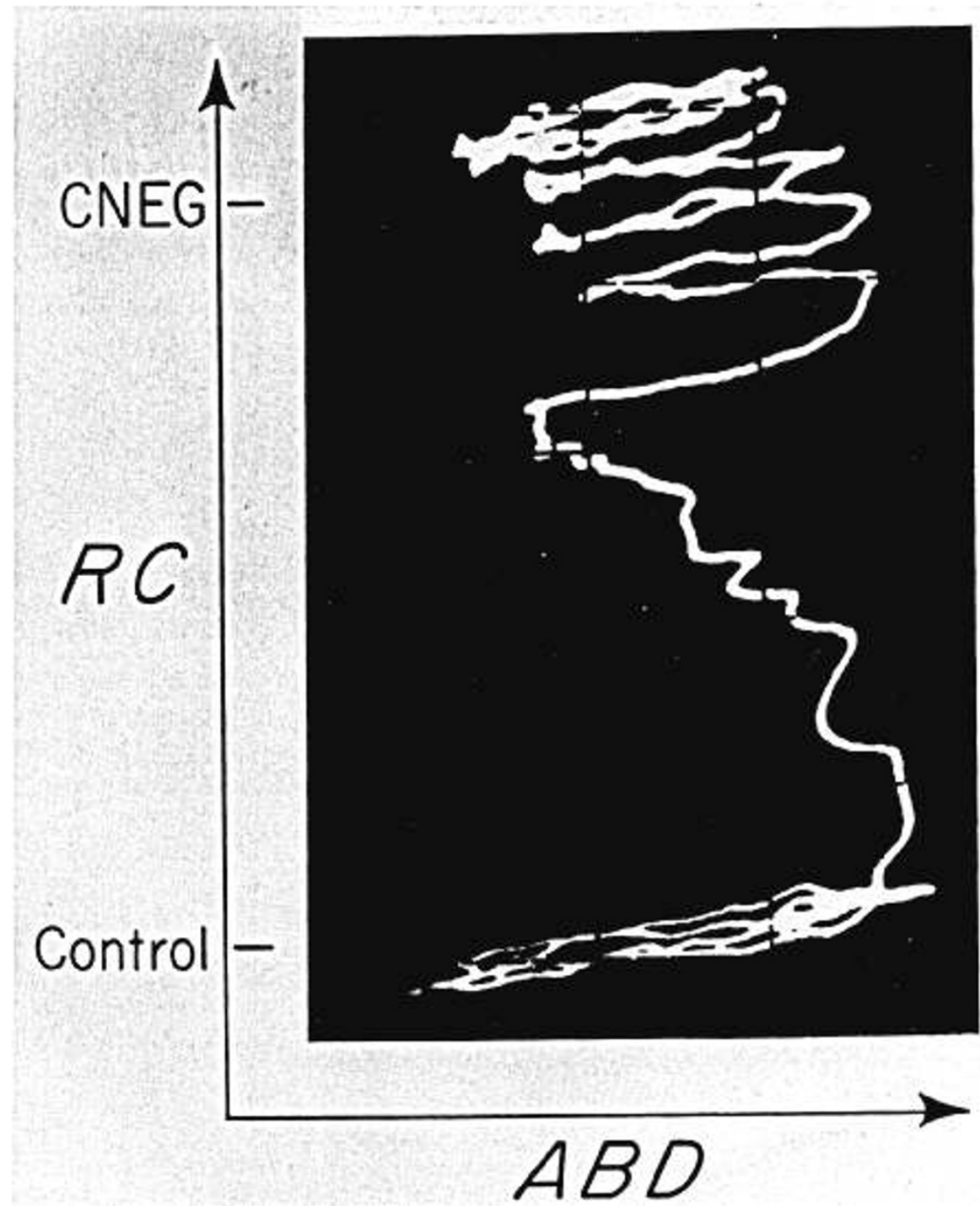

Fig. 2. Recording of rib cage (RC) and abdominal (ABD) A-P magnetometers during control breathing (bottom loops) and at increased lung volume (top loops) in a full term infant.

analyzed in an X-Y representation, was labeled "in-phase" movement of rib cage and abdomen. These authors suggested that the infant rib cage, being more compliant than that of the adult, might undergo displacement with more than one degree of freedom (i.e., become distorted). Knill et al. (17) reported distortion of the rib cage in infants during active sleep, whereas, during quiet sleep, the rib cage moved as a unit, with minimal distortion. They suggested that the degree of distortion of the rib cage was inversely related to the activity of the intercostal muscles.

Our observations are consistent with the previous work. We find that the rib cage moves substantially as a unit, with one degree of freedom during spontaneous breathing and during most occluded inspiratory efforts when the infant is apparently in quiet sleep. However, we also observed that, on occasion, the rib cage appears to move with more than one degree of freedom during spontaneous breathing as well as during occlusion. We believe that these different patterns of rib cage movement are most likely due to different patterns of activity of the rib cage musculature. Accordingly, we have not tried to assign a unique volume calibration to the movement of the rib cage detected at a single site on the anterior-posterior plane.

The decrease in ventilation found in the full term infants during CNEG is similar to that observed by Bancalari et al. (1) in premature infants with hyaline membrane disease. In our infants with normal lungs, the decrease in ventilation was due entirely to a decrease in respiratory frequency, while both frequency and tidal volume were reduced in the subjects of Bancalari et al. Our premature infants showed no consistent ventilatory response to breathing at increased lung volume. Ventilation decreased in three of the premature infants, associated with a decrease in respiratory frequency. The fact that ventilation and frequency were less consistently affected by increasing lung volume may be because smaller increases in lung volume occurred in the premature infants. In all infants in whom frequency decreased, the decrease was the result of prolongation of expiration. These effects on ventilation are similar to those reported by Saunders et al. (24), whose subjects were largely normal, premature infants.

The decrease in respiratory rate is presumably due to stimulation of pulmonary stretch receptors. Previous studies of the Hering-Breuer reflex in infants have been limited to measurement of the duration of apnea after lung inflation (5), or to comparison of inspiratory duration before and after airway occlusion $(21,26)$. None of these studies has been concerned with the effects of changing lung volume on respiratory rate after the first breath. Martin et al. (20) have shown a similar decrease in respiratory rate during positive pressure breathing in infants. This decrease was also due to prolongation of expiration.

Mask occlusion pressure depends on the geometry of the inspiratory muscles. Because increases in lung volume can change the configuration of these muscles, the inspiratory pressure developed 


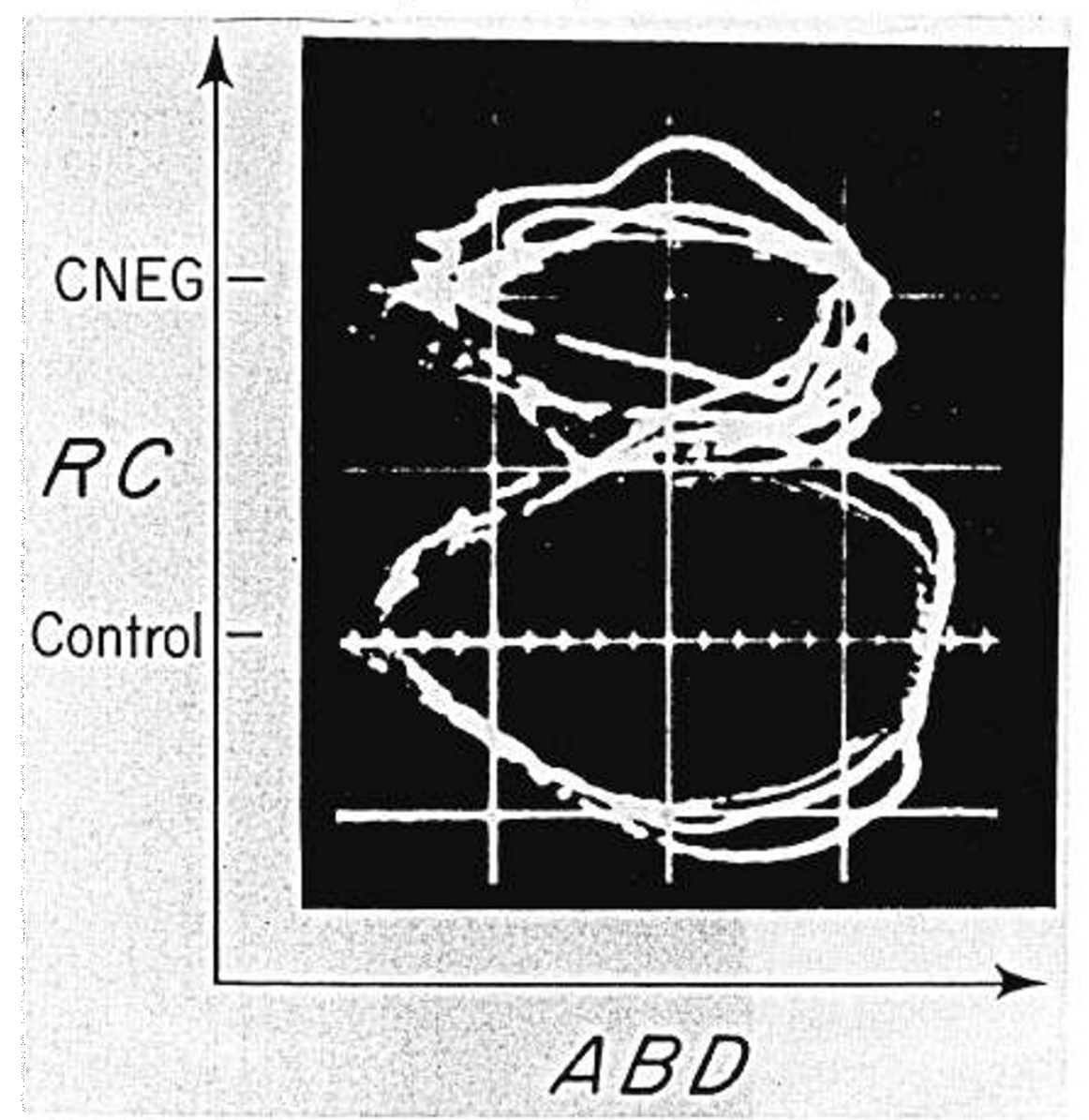

Fig. 3. A-P magnetometer recordỉng in a premature intant betore and atter an increase in lung volume.

$\underset{\substack{\text { Pressure } \\ \text { CmH }}}{\text { Box }-10}$

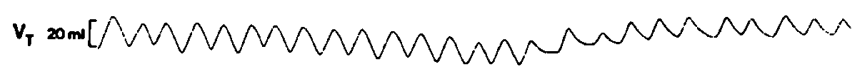

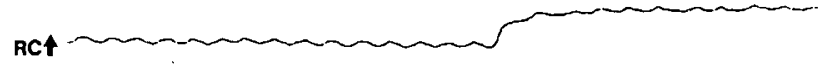

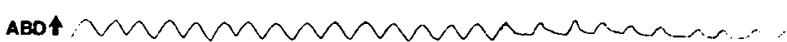

Fig. 4. Tidal volume $\left(\mathrm{V}_{\mathrm{r}}\right)$ and magnetometer recording of a premature infant during an increase in lung volume when the distending pressure (CNEG) was turned on. Arrows indicate the direction of increasing diameter.

during occlusion might then be decreased for two reasons. First, the force developed by a contracting muscle decreases as its length decreases (6). Second, because of the Laplace relationship, as the diaphragm becomes flatter and, therefore, increases its radius of curvature, its mechanical advantage in converting tension to pressure is diminished. This has been observed in both animals (19) and adult man (22).

Preliminary reports of several studies have shown increases in lung volume to affect occlusion pressure in adults. Sabbagh et al. (23) demonstrated an inverse linear relationship between occlusion pressure and resting functional residual capacity (FRC) in conscious adults breathing room air. Similarly, Shaffer et al. (25) showed that adult subjects with larger FRC values have lower occlusion pressures at a fixed level of minute ventilation than those with smaller FRC's. It is likely that lung volume increases in these subjects compromised the configuration of the inspiratory muscles so that occlusion pressure was lower at a given level of respiratory drive.

In contrast to studies in adults, mask occlusion pressure in our infants was not affected in a consistent manner when lung volume was increased, but, in most infants, any changes seen were small. This can be explained if the mechanical advantage of the pertinent inspiratory muscles was defended. This would occur either if expiratory muscle activity prevented an increase in lung volume, or if the configuration of the pertinent inspiratory muscles was maintained after the increase in lung volume. We have shown in newborn infants that an increase in lung volume was achieved with CNEG, and was accounted for largely by rib cage expansion with little abdominal displacement.

We may expect that rib cage expansion will be associated with a decrease in the mechanical advantage of inspiratory rib cage musculature and, therefore, that if these muscles were the primary determinant of occlusion pressure, we could anticipate a fall in $\mathrm{P}_{m}$ with CNEG. We did not observe such a fall. We suggest then that rib cage muscles are not the primary determinant of occlusion pressure.

In contrast, the absence of a change in end-expiratory abdominal A-P diameter in the face of a distending pressure applied to the entire body surface below the neck implies that some increase in abdominal expiratory muscle activity must have occurred at end-expiration during CNEG. This is consistent with the increased abdominal muscle activity seen in animals during application of continuous distending pressure $(2,3)$. Furthermore, if changes in A-P diameter of the abdomen reflect displacement of the diaphragm in infants, then we suggest that the end-expiratory abdominal muscle activity inferred from our observations (Fig. 2-5) 


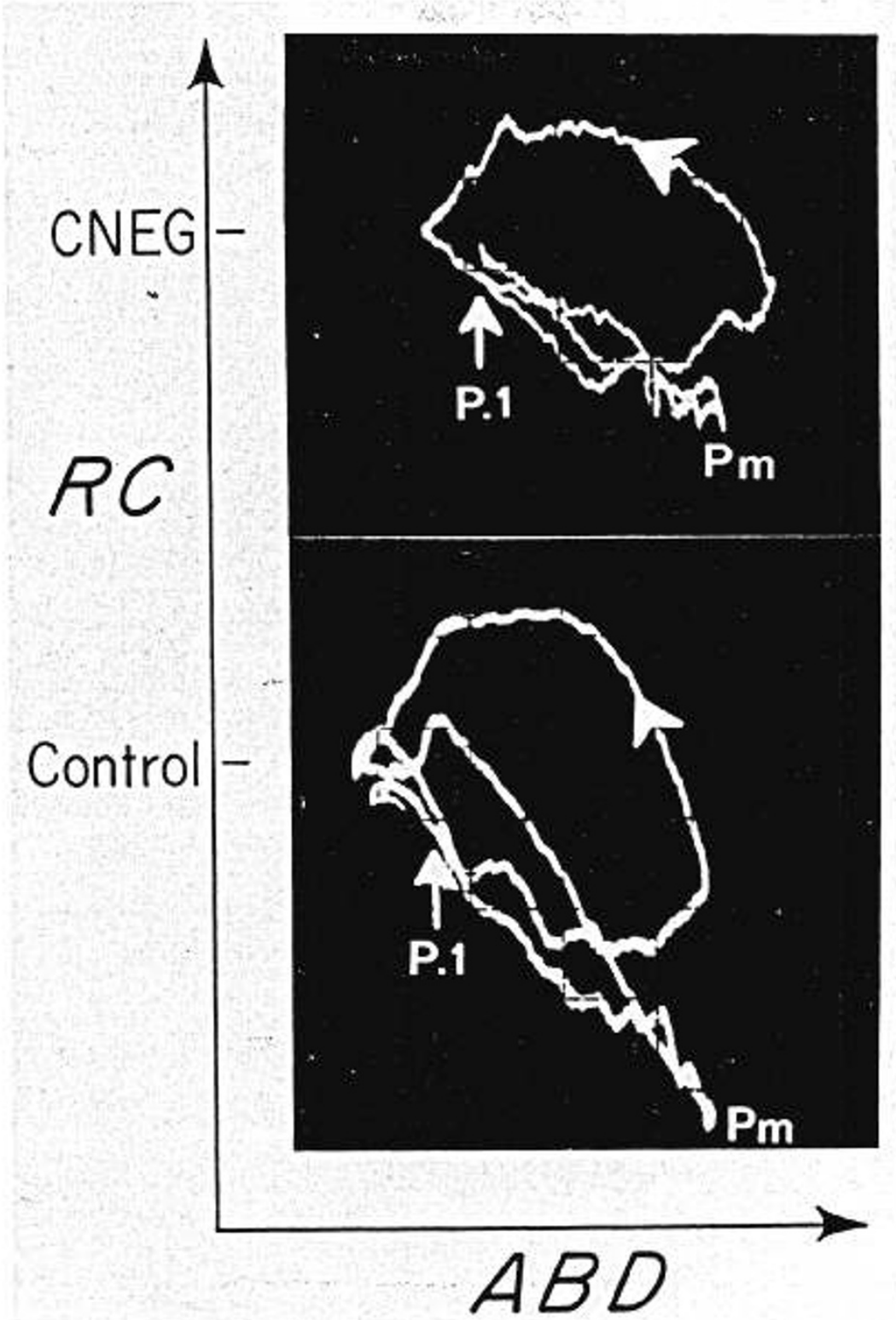

Fig. 5. Magnetometer recording of the tidal volume loops and isovolume maneuvers on and off CNEG. The direction of the tidal loops is indicated, as is the rib cage-abdomen configuration where $P_{.}$and $P_{m}$ were measured. Note that the abdominal diameter is similar on and off CNEG at $P_{\text {., and }} P_{m}$ while the rib cage has expanded.

helps to maintain the resting configuration and, thus, the mechanical advantage of the diaphragm, although at the expense of the rib cage inspiratory muscles.

Previous studies have shown that an increase in lung volume does not compromise the configuration of all the inspiratory muscles to the same degree. Goldman and coworkers $(10,11,13$, 14), showed in adults that transdiaphragmatic pressure at a given level of diaphragm electrical activity was diminished relatively little when the rib cage accounted for an increase in lung volume. In contrast, transdiaphragmatic pressure was markedly reduced if the increase in lung volume was associated with outward displacement of the abdominal wall.

It is apparent from the magnetometer measurements that the configuration of the diaphragm (as assessed by abdominal A-P diameter) was similar during inspiration whether the airway was occluded or not, and regardless of end-expiratory lung volume. The fact that mask occlusion pressure changed little in most infants suggests that the diaphragm is the primary muscle which determines $P_{m}$. However, we do not suggest that the diaphragm is the only determinant of $\mathrm{P}_{\mathrm{m}}$. The relaxation of abdominal expira- tory muscle activity at the beginning of inspiration will contribute to the development of $P_{m}$. In addition, we may expect that the inspiratory muscles of the rib cage will contribute to $\mathrm{P}_{\mathrm{m}}$. Furthermore, we have indicated that some of the variability in $P_{m}$ reflects different patterns of rib cage distortion during occlusion.

In spite of the variable effect on occlusion pressure, CNEG resulted in a consistent pattern of abdominothoracic displacements in the infants studied. The neuromuscular response of the respiratory system to distending pressure may differ among species, and may further depend on age, neural development, and level of consciousness. The overall response may be either largely expiratory excitation, presumably vagally-mediated, or inspiratory excitation, possibly mediated through diaphragmatic tension (10, $15)$, or in the conscious individual, a choice between these alternatives. We suggest that the relative constancy of occlusion pressure observed in our study during an increase in FRC related to the level of vagal activity and increased abdominal muscle activity in these infants.

Our measurements were made during periods of quiet, regular breathing, suggesting that the infants were in quiet, or nonrapid 
eye movement sleep. However, due to the length of the studies and the maneuvers carried out, it is likely that change of sleep state occurred. Although Knill et al. (17) reported that compensation by infants to a respiratory elastic load was less during active than during quiet sleep, the data of Frantz et al. (7) indicate that the mask pressure of the first occluded breath is similar in both sleep states. Because we observed the effect of lung volume changes on the mask occlusion pressure of only the first occluded breath, changes in sleep state should not have affected our measurements. Using magnetometers, Knill et al. (17) showed that end-expiratory abdominal displacement for a given tidal volume changed with sleep state. In our study, neither end-inspiratory nor end-expiratory abdominal diameter changed with increases in lung volume.

\section{CONCLUSION}

We have observed the effects on respiration of increasing lung volume by continuous distending pressure in newborn infants. Minute ventilation and respiratory frequency decreased in full term infants, but there was no consistent change in ventilation in the premature infants. In both the full term and premature infants, there were variable changes in occlusion pressure at increased lung volume. In both groups, the increase in lung volume was accounted for largely by expansion of the rib cage so that the configuration of the diaphragm and its mechanical advantage were maintained. These results suggest that the diaphragm is the major determinant of mask occlusion pressure in infants.

\section{REFERENCES AND NOTES}

1. Bancalari, E., Garcia, O. L., and Jesse, M. J.: Effects of continuous negative pressure on lung mechanics in idiopathic respiratory distress syndrome. Pediatrics, 51: 485 (1973).

2. Bishop, B., and Bachofen, $\mathrm{H}$.: Vagal control of ventilation and respiratory muscles during elevated pressures in the cat. J. Appl. Physiol., 32: 103 (1972).

3. Bruce, E. N., Gillespie, J. R., Goldman, M. D., and Mead, J.: Responses of unanesthetized monkeys to continuous positive-pressure breathing (CPPB). Fed. Proc., 35: 633 (1976).

4. Cosgrove, J. F., Neuburger, N., Bryan, M. H., Bryan, A. C., and Levison, H.: A new method of evaluating the chemosensitivity of the respiratory center in children. Pediatrics, 56: 972 (1975).

5. Cross, K. W., Klaus, M., Tooley, W. H., and Weisser, K.: The response of the newborn baby to inflation of the lungs. J. Physiol., 15I: 551 (1960).

6. Evans, C. L., and Hill, A. V.: The relation of length to tension development and heat production on contraction in muscle. J. Physiol., 49: 1 (1914).

7. Frantz, I. D., Adler, S. M., Abroms, I. F., and Thach, B. T.: Respiratory response to airway occlusion in infants: sleep state and maturation. J. Appl. Physiol., 41: 634 (1977).

8. Frantz, I. D., Adler, S. M., Thach, B. T., and Taeusch, H. W.: Maturational effects on respiratory responses to carbon dioxide in premature infants. J. Appl. Physiol., 41: 41 (1976).

9. Goldman, M. D.: Mechanical factors in the regulation of breathing pattern and FRC. Bull. Physio.-Pathol. Respir., 11: 98 (1975).
10. Goldman, M. D., Bruce, E. N., Loh, L., and Mead, J.: Changes in diaphragm activation with posture during rebreathing. Fed. Proc., 34: 371 (1975).

11. Goldman, M. D.. Grassino. A. E., Mead. J.. and Sears. T. A.: Pressure-volume characteristics of the diaphragm during voluntary contraction. Fed. Proc., 31: 321 (1972).

12. Goldman, M. D., Grassino, A., Mead, J., and Sears, T. A.: Mechanics of the human diaphragm during voluntary contraction: dynamics. J. Appl. Physiol. 44: 840 (1978).

13. Grassino, A. E., Goldman, M. D., and Mead, J.: Influence of chest wall configuration on the static and dynamic characteristics of the contracting diaphragm. In: L. D. Pengelly, A. S. Rebuck, and E. J. M. Campbell: Loaded Breathing. p. 50 (Livingstone, Edinburgh and London, 1974).

14. Grassino, A., Goldman, M. D., Mead, J., and Sears, T. A.: Mechanics of the human diaphragm during voluntary contraction: statics. J. Appl. Physiol. 44: 829 (1978).

15. Green, M., Mead, J., and Sears, T. A.: Effects of loading on respiratory muscle control in man. In: L. D. Pengelly, A. S. Rebuck, and E. J. M. Campbell: Loaded Breathing, p. 73 (Livingstone, Edinburgh and London, 1974).

16. Kattwinkel, J., Nearman, H. S., Fanaroff, A. A., Katona, P. G., and Klaus, M. H.: Apnea of prematurity. J. Pediatr., 86: 588 (1975).

17. Knill, R., Andrews, W., Bryan, A. C., and Bryan, M. H.: Respiratory load compensation in infants. J. Appl. Physiol., 40: 357 (1976).

18. Konno, K., and Mead, J.: Static volume-pressure characteristics of the rib cage and abdomen. J. Appl. Physiol., 24: 544 (1968)

18. Marshall, R.: Relationships between stimulus and work of breathing at different lung volumes. J. Appl. Physiol., 17: 917 (1962).

20. Martin, R. J., Okken, A., Katona, P., and Klaus, M.: Evidence for a tonic component of the Hering-Breuer inflation reflex in the term neonate. Pediatr. Res. 11: 575 (1977).

21. Olinsky, A., Bryan, M., and Bryan, A.: The influence of lung inflation on respiratory control in neonates. J. Appl. Physiol., 36: 426 (1974).

22. Pengelly, L. D., Adlerson, A. M., and Milic-Emili, J.: Mechanics of the diaphragm. J. Appl. Physiol., 30: 797 (1971).

23. Sabbagh, H., Evanich, M. J., Lourenco, R. V., and Lopata, M.: Factors affecting mouth occlusion pressure in conscious man. Clin. Res., 23: 504A (1975).

24. Saunders, R. A., Milner, A. D., and Hopkin, I. E.: The effects of continuous positive airway pressure on lung mechanics and lung volume in the neonate. Biol. Neonate, 29: 178 (1976).

25. Shaffer, T. H., Altose, M. D., Lederer, D. H., and Cherniack, N. D.: The interaction of FRC and ventilation on occlusion pressure: theorectical and experimental results for conscious man. Am. Rev. Respir. Dis. 1/3: 195 (1975).

26. Taeusch, H. W., Carson, S., Frantz, I. D., and Milic-Emili, J.: Respiratory regulation after elastic loading and $\mathrm{CO}_{2}$ rebreathing in normal term infants. J. Pediatr., 88: 102 (1976).

27. Thibeault, D. W., Wong, M. M., and Auld, P. A. M.: Thoracic gas volume changes in premature infants. Pediatrics, 40: 403 (1967).

28. Whitelaw, W. A., Derenne, J. Ph., and Milic-Emili, J.: Occlusion pressure as a measure of respiratory center output in conscious man. Respir. Physiol., 23: 181 (1975).

29. The authors thank the nurses and physicians of the Special Care Nursery. Boston Hospital for Women, for their help and support, and Drs. M. E. Avery, V. Chernick, H. W. Taeusch, and J. Mead for their comments on the manuscript.

30. This research was supported, in part, by the Charles H. Hood Foundation. Dr. Stark is an E. L. Trudeau Fellow of the American Lung Association. Dr. Frantz is the recipient of a Young Investigator Pulmonary Research Grant from the National Heart and Lung Institute, National Institutes of Health. Dr. Goldman is supported, in part, by National Institutes of Health Grant HL 00256.

31. Requests for reprints should be addressed to: Dr. Ann R. Stark, Joint Program in Neonatology, 221 Longwood Avenue, Boston, MA 02115 (USA).

32. Received for publication December 20, 1977.

33. Accepted for publication May 15, 1978 . 